\title{
ON SOME EXTENSIONS OF THE ACCELERATED OVERRELAXATION (AOR) THEORY
}

\author{
A. HADJIDIMOS and A. YEYIOS \\ Department of Mathematics \\ University of Ioannina \\ Ioannina, GREECE
}

(Received June 5, 1980 and in revised form March 30, 1981)

ABSTRACT. This paper extends the convergence theory of the Accelerated Overrelaxation (AOR) method to cases analogous to those considered first by Ostrowski and then by Varga in connection with the Successive Overrelaxation (SOR) method. Among others, the Ostrowski Theorem, some of the theorems by Varga on the extensions of the SOR theory, and some recent results by Niethammer and by the authors are obtained as special cases of the work presented in this paper. In addition, several points are raised which suggest further research.

KEY WOROS AND PHRASES. Three-part splittings, First order iterative method, Second order iterative method, Successive Overrelaxation (SOR) method, Extrapolation, Accelerated Overrelaxation (AOR) method.

1980 MATHEMATICS SUBJECT CLASSIFICATION CODE. Primary $65 F 10$.

1. INTRODUCTION AND PRELIMINARIES.

In a series of papers, Sisler [1]-[3] conceived and studied the idea of twoparametric three-part splittings of the matrix A for the numerical solution of the linear system

$$
A x=b
$$

by a first order iterative method. His method was, in fact, an Extrapolation of the Successive Overrelaxation ( $\mathrm{SOR}$ ) one. Sisler's work has been extended recently by Niethammer [4]. A couple of years ago, Hadjidimos [5] introduced an equivalent splitting which led to the Accelerated Overrelaxation (AOR) method. This idea was exploited further and some interesting results were obtained (see [6], [7] [8], 
[9] and [10]). Under the assumptions that A, in (1.1), is nonsingular with nonvanishing diagonal elements, the AOR method (scheme) is the following

$$
\begin{aligned}
x^{(m+1)}=\left(D-r A_{L}\right)^{-1}\left[(1-\omega) D+(\omega-r) A_{L}+w A_{U}\right] x^{(m)} & +w\left(D-r A_{L}\right)^{-1} b \\
& m=0,1,2, \ldots,
\end{aligned}
$$

where $D=\operatorname{diag}(A),-A_{L}$ and $-A_{U}$ are the strictly lower and upper triangular parts of $A$, and $\omega(\neq 0)$ and $r$ are the overrelaxation and acceleration parameters. It is pointed out that, for $\mathrm{r} \neq 0$, (1.2) becomes an Extrapolated SOR method, with extrapolation parameter $\omega / r$ and overrelaxation parameter $r$. It was under this restriction that both Sisler [1] - [3] and Niethammer [4] considered and studied the method (1.2).

Now we assume that the $\mathrm{n} \times \mathrm{n}$ matrix $\mathrm{A}$ is Hermitian and can be split into the form

$$
A=D-E-E^{H} \text {, }
$$

where $D$ and $E$ are $n \times n$ matrices, $D$ is Hermitian and positive definite, and $E^{H}$ is the complex conjugate transpose of E. Based on the splitting (1.3), we generalize the scheme (1.2) as follows

$$
\mathrm{x}^{(\mathrm{m}+1)}=\mathrm{L}_{\mathrm{r}, \omega} \mathrm{x}^{(\mathrm{m})}+\omega(\mathrm{D}-\mathrm{rE})^{-1} \mathrm{~b} \quad \mid \mathrm{m}=0,1,2, \ldots,
$$

where the iteration matrix of the procedure is

$$
\mathrm{L}_{\mathrm{r}, \omega}=(\mathrm{D}-\mathrm{rE})^{-1}\left[(1-\omega) \mathrm{D}+(\omega-\mathrm{r}) \mathrm{E}+\omega \mathrm{E}^{\mathrm{H}}\right] \text {; }
$$

for the existence of $(1.4)$ and (1.5), $\operatorname{det}(D-r E) \neq 0$ is assumed. In (1.4), $D$ and E need not be diagonal and strictly lower triangular respectively, as the matrices $D$ and $A_{L}$ in (1.2) have to be. It is also noted that, for $r=\omega$ and $D$ being either the diagonal or a block diagonal part of A, the method (1.4) becomes the well known generalized SOR method for which an important convergence theorem was given by Ostrowski [11] (see also Varga [12], p. 77). The corresponding theory was extended by Varga [13], who also suggested that the most general form for $E$ is

$$
E=\frac{1}{2}(D-A+S),
$$

with $\mathrm{S}$ being any skew-Hermitian matrix. Following the steps of the extension by Varga, Hadjidimos [14] considered and studied the case where D is negative definite and found some interesting results. 
The purpose of this paper is to extend the convergence theory by Ostrowski and Varga [12, 13] so as to cover the case of the generalized AOR scheme (1.4) and to raise questions for further research in this area. This is what we do in the next two sections; in addition, some comparisons and comments relating recent results by de Pillis [15], Niethammer [4], Neumann and Varga [16], and the present authors with those of this paper are made.

\section{BASIC CONVERGENCE THEORY OF THE AOR METHOD (1.4).}

We begin our analysis by giving five Lemmas on which our theory is based.

LEMMA 1. Let $A=D-E-E^{H}$ be an $n \times n$ Hermitian matrix, where $D$ is Hermitian and positive definite. Then the eigenvalues of the generalized Jacobi matrix $B \equiv D^{-1}\left(E+E^{H}\right) \equiv I-D^{-1} A$ ( $I$ is the unit matrix of order $n$ ) are real.

PROOF. Let $\mathrm{D}^{\frac{1}{2}}$ be the unique Hermitian positive definite square root matrix of the Hermitian positive definite matrix D (see [17], pp. 22-24). We form the matrix $\hat{B}$ as follows

$$
\hat{B}=D^{\frac{1}{2}} B D^{-\frac{1}{2}}=D^{-\frac{1}{2}}\left(E+E^{H}\right) D^{-\frac{1}{2}} \text {. }
$$

The last expression shows that $\hat{B}$ is Hermitian and therefore it possesses real eigenvalues. So does its similar matrix B.

LEMMA 2. Under the hypotheses of Lemma 1 , let $\mu_{i} \mid i=1(1) \mathrm{n}$ be the eigenvalues of $B$. Then $A$ is positive definite iff $\mu_{i}<1 \quad i=1(1) \mathrm{n}$.

PROOF. From Lemma 1 , we have $I-B=D^{-1} A$. Thus, the real number $1-\mu_{i}$ $\mathrm{I}=1(1) \mathrm{n}$ are the eigenvalues of the product $\mathrm{D}^{-1} \mathrm{~A}$ where the matrices $\mathrm{D}^{-1}$ and $\mathrm{A}$ are Hermitian with $\mathrm{D}^{-1}$ positive definite. Then, according to Wigner [18], $\mathrm{A}$ is positive definite iff $1-\mu_{i}>0 \quad \mid i=1(1) n$, which proves our assertion.

LEMMA 3. Under the hypotheses of Lemma 1, the matrix M below

$$
M \equiv\left(\frac{2-\omega}{\omega}\right) D+\left(\frac{\omega-r}{\omega}\right)\left(E+E^{H}\right) \equiv\left(\frac{2-r}{\omega}\right) D+\left(\frac{r-\omega}{\omega}\right) A,
$$

is positive definite iff the matrix

$$
\hat{M} \equiv\left(\frac{2-\omega}{\omega}\right) I+\left(\frac{\omega-r}{\omega}\right) \hat{B}
$$

is positive definite.

PROOF. Using the definitions (2.3), (2.1), and (2.2), we obtain 


$$
M=\left(\frac{2-\omega}{\omega}\right) I+\left(\frac{w-r}{\omega}\right) D^{-\frac{1}{2}}\left(E+E^{H}\right) D^{-\frac{1}{2}}=D^{-\frac{1}{2}} M D^{-\frac{1}{2}}=D^{-\frac{1}{2}} M\left(D^{-\frac{1}{2}}\right)^{H} .
$$

In view of the last expression for $\hat{\mathrm{M}}$ and according to Corollary 2.8 (p. 24 of [17]), the lemma is proved.

LEMMA 4. Under the hypotheses of Lemmas 1 and 2 , let $\mu_{\mathrm{m}}=\min \mu_{i}$ and $\mu_{M}=\max _{i} \mu_{i}$. Then the matrix $\hat{M}$ in $(2.3)$ is positive definite iff the parameters $\omega(\neq 0)$ and $r$ take any values from their domains, as these are defined and given in the table in each specific case which depends on the relative position of $\mu_{m}$ and $\mu_{M}$ with respect to (wrt) zero.

PROOF. The eigenvalues $\mu_{i} \mid i=1(1)$ n of $B$ are the same as those of $\hat{B}$ (Lemma 1). Therefore, according to Lemma 3, the Hermitian matrix $\hat{M}$ (and consequently M) is positive definite iff all its eigenvalues given, because of (2.3), by the expressions

$$
\left[(2-\omega)+(\omega-r) \mu_{i}\right] / \omega \quad \mid i=1(1) n,
$$

are positive. Now we have to distinguish six cases, depending on the relative position of $\mu_{m}$ and $\mu_{M}$ wrt zero. These are given in the table. In what follows, we work out only Case 1 (the others can be treated similarly). Assume then that $0<\mu_{\mathrm{m}}<\mu_{\mathrm{M}}$. Thus, if $\omega<0$, the relationships $\left[(2-\omega)+(\omega-r) \mu_{i}\right] / \omega>0$ $\mid i=1(1) n$ lead to the equivalent ones $\omega+(2-\omega) / \mu_{i}<r \mid i=1(1) n$ which, in turn, are equivalent to $\omega+(2-\omega) / \mu_{m}<r$. If, on the other hand, $\omega>0$, we arrive at $\omega+(2-\omega) / \mu_{i}>r \mid i=1(1) n$. Now we have to consider three cases. If $\omega<2$, it is implied that $r<\omega+(2-\omega) / \mu_{M}$; if $\omega=2$, then $r<2$; if $\omega>2$, $r<\omega+(2-\omega) / \mu_{m}$. These results, together with those obtained from the other five cases, given in the table, prove the present lemma. 
Table

giving the possible domains of the parameters $\omega$ and $r$

\begin{tabular}{|c|c|c|c|}
\hline Case & $\begin{array}{l}\text { Relative position } \\
\text { of } \mu_{m} \text { and } H_{M} \text { wrt zero }\end{array}$ & $\omega$-domain & r-domain \\
\hline \multirow{4}{*}{1} & \multirow{4}{*}{$0<\mu_{m} \leqq \mu_{M}$} & $(-\infty, 0)$ & $\left(\omega+(2-\omega) / \mu_{m},+\infty\right)$ \\
\hline & & $(0,2)$ & $\left(-\infty, \omega+(2-\omega) / \mu_{M}\right)$ \\
\hline & & 2 & $(-\infty, 2)$ \\
\hline & & $(2,+\infty)$ & $\left(-\infty, \omega+(2-\omega) / \mu_{m}\right)$ \\
\hline 2 & $0=\mu_{\mathrm{m}}<\mu_{\mathrm{M}}$ & $(0,2)$ & $\left(-\infty, \omega+(2-\omega) / \mu_{M}\right)$ \\
\hline 3 & $0=\mu_{\mathrm{m}}=\mu_{\mathrm{M}}$ & $(0,2)$ & $\left(-\infty,++^{\infty}\right)$ \\
\hline 4 & $\mu_{m}<0<\mu_{M}$ & $(0,2)$ & $\left(\omega+(2-\omega) / \mu_{m}, \omega+(2-\omega) / \mu_{M}\right)$ \\
\hline 5 & $\mu_{\mathrm{m}}<\mu_{\mathrm{M}}=0$ & $(0,2)$ & $\left(\omega+(2-\omega) / \mu_{m},+\infty\right)$ \\
\hline \multirow{4}{*}{6} & \multirow{4}{*}{$\mu_{m} \leqq \mu_{M}<0$} & $(-\infty, 0)$ & $\left(-\infty, \omega+(2-\omega) / \mu_{M}\right)$ \\
\hline & & $(0,2)$ & $\left(\omega+(2-\omega) / \mu_{m},+\infty\right)$ \\
\hline & & 2 & $(2,+\infty)$ \\
\hline & & $(2,+\infty)$ & $\left(\omega+(2-\omega) / H_{M},+\infty\right)$ \\
\hline
\end{tabular}

COROLLARY. Under the assumptions of Lemma 4 and the additional assumptions that $D=\operatorname{diag}(A)$ or $D$ is a block diagonal part of $A$ and in either case $D$ is positive definite, then $\hat{M}$ (and consequently $M$ ) is positive definite iff the $\omega$ - and $r$-domains are those given in the Cases 3 and 4 of the table (see also [10] for the case $D=\operatorname{diag}(A))$.

PROOF. Since $D=\operatorname{diag}(A)$ or $D$ is a block diagonal part of $A$, we have from the expression for $B$ (and therefore $\hat{B}$ ) that $\operatorname{tr}(B)=0$. The latter implies that either $\mu_{m}=\mu_{M}=0$ or $\mu_{m}<0<\mu_{M}$ so that only the Cases 3 and 4 of the table can occur. This completes the proof. 
LEMMA 5. (Householder-John Theorem [19]). Under the hypotheses of Lemma 1, let $A=A_{1}-A_{2}$ be a splitting of A generating the first order scheme $x^{(m+1)}=$ $A_{1}^{-1} A_{2} x^{(m)}+A_{1}^{-1} b \mid m=0,1,2, \ldots$ for the numerical solution of (1.1). If $A_{1}^{H}+A_{2}$ is positive definite, then $\rho\left(A_{1}^{-1} A_{2}\right)<1$ iff $A$ is positive definite.

THEOREM 1. (Generalized Ostrowski Theorem). Let $\mathrm{A}=\mathrm{D}-\mathrm{E}-\mathrm{E}^{\mathrm{H}}$ be an $\mathrm{n} \times \mathrm{n}$ Hermitian matrix, where $D$ is Hermitian and positive definite and the $\operatorname{det}(D-r E) \neq 0$ for any permissible pair of parameters $r, \omega$ from the table depending on the position of $\mu_{m}$ and $\mu_{M}$ wrt zero. Then, for the parameters $r, \omega$ from the appropriate intervals of the table, the AOR method $(1.4)$ converges $\left(\rho\left(\mathrm{L}_{\mathrm{r}, \omega}\right)<1\right)$ iff A is positive definite.

PROOF. It is obvious that, in our case of the scheme (1.4), the splitting of Lemma 5 is defined by $A_{1} \equiv \frac{1}{\omega}(D-r E)$ and $A_{2} \equiv \frac{1}{\omega}\left[(1-\omega) D+(\omega-r) E+\omega E^{H}\right]$. Hence, $A_{1}^{H}+A_{2}=M$. Since any pair $(r, \omega)$ of the parameters is taken so that it belongs to the appropriate intervals of the table, then according to Lemmas 3 and 4 the Hermitian matrix $M$ is positive definite. Thus by virtue of Lemma 5 , the present theorem is proved.

REMARK. It is obvious that the most important part of Theorem 1 is that where the positive definiteness of A implies convergence of the AOR scheme. The converse is of theoretical value only, unless knowledge of the position of $\mu_{m}$ and $\mu_{M}$ wrt zero is known in advance as e.g. in the cases of the Corollary of Lemma 4 . If we have to know the exact values for $\mu_{m}$ and $\mu_{M}$ in order to be able to form the $\omega$ - and r-domains from the table and find that $\rho\left(\mathrm{L}_{\mathrm{r}, \omega}\right)<1$ for any permissable pair $(\mathrm{r}, \omega)$ so that positive definiteness for $A$ is implied, then we can reach the last conclusion straightforwardly from the value of $\mu_{M}$ which must be less than one (see Lemma 2).

THEOREM 2. Let $A=D-E-E^{H}$ be an $n \times n$ Hermitian positive definite matrix, where $D$ is Hermitian and the $\operatorname{det}(D-r E) \neq 0$ for one pair of parameters $r, \omega$. If $\lambda_{j} \mid j=1(1) n$ are the eigenvalues of $L_{r, \omega}$ and $u_{j} \mid j=1(1) n$ the corresponding eigenvectors, which may not be all independent, then the AOR method (1.4) converges $\left(\rho\left(L_{r, \omega}\right)<1\right)$ iff $u_{j}^{H} M_{j}>0 \quad \mid j=1(1) n$. 
PROOF. We follow the basic steps of the analysis in [13] and begin with the relationships

$$
L_{r, \omega} u_{j}=\lambda_{j} u_{j} \quad \mid j=1(1) n \text {. }
$$

By using (1.3), (1.5), (1.6), and notation similar to that in [14] giving that

$$
a_{j}=u_{j}^{H} A u_{j}>0, \quad d_{j}=u_{j}^{H} D u_{j}, \quad i \sigma_{j}=u_{j}^{H} u_{j} \quad \mid j=1(1) n,
$$

with $\sigma_{j} \in \mathbb{R}$ and $i=(-1)^{\frac{1}{2}}$, we can obtain that

$$
\lambda_{j}=\frac{\left[(2-r) d_{j}-(2 \omega-r) a_{j}\right]-i r \sigma_{j}}{\left[(2-r) d_{j}+r a_{j}\right]-i r \sigma_{j}} \quad \mid j=1(1) n .
$$

From (2.5) and by virtue of (2.2) and (2.4), it can be taken that

$$
\left|\lambda_{j}\right|^{2}=1-\frac{4 \omega^{2} a_{j} u_{j}^{\mathrm{Mu}_{j}}}{\left[(2-r) d_{j}+r a_{j}\right]^{2}+r^{2} \sigma_{j}^{2}} \quad j=1(1) n \text {. }
$$

It is obvious that relationships (2.6) prove the theorem.

REMARK. We observe that, if $M$ is positive definite, convergence of the $A O R$ method (1.4) is guaranteed under the assumptions of Theorem 2. Especially for the SOR method $(r=\omega)$, we have that $M=\left(\frac{2-\omega}{\omega}\right) D$. Thus: $\left.i\right)$ the part of Theorem 1 of Varga [13] "for any D positive definite and any $\omega \in(0,2) \rho\left(L_{\omega, \omega}\right)<1$ " and ii) the part of Theorem 2 of Hadjidimos [14] "for any D negative definite and $\omega \in(-\infty, 0) U$ $(2,+\infty)$ " become special cases of the previous Theorem 2 . Consequently, convergence of these extensions of the SOR method is implied straightforward. In view of the observation made at the beginning of this Remark, sufficient conditions for the convergence of the AOR method can be given in the following two Corollaries.

COROLLARY 1. Let $\mathrm{A}=\mathrm{D}-\mathrm{E}-\mathrm{E}^{\mathrm{H}}$ be an $\mathrm{n} \times \mathrm{n}$ Hermitian positive definite matrix and $D$ be Hermitian and positive definite. Then sufficient conditions for the AOR method to converge are $\operatorname{det}(D-r E) \neq 0$ and $0<\omega \leqq r \leqq 2$, $\omega \neq 2$ (see also [10])

PROOF. We observe that the assumptions of Theorem 2 are satisfied. However, the restrictions on $r$ and $\omega$ give that $\frac{2-r}{\omega} \geq 0$ and $\frac{r-\omega}{\omega} \geq 0$, where at least one of the inequalities must be a strict one. From these relationships, the fact that D and $A$ are Hermitian and positive definite, and the second expression for the matrix 
$M$ in (2.2), we readily deduce that $M$ is positive definite. Hence, the AOR method converges.

COROLlARY 2. Let $A=D-E-E^{H}$ be an $n \times n$ Hermitian positive definte matrix and $\mathrm{D}$ be Hermitian and negative definite. Then sufficient conditions for the AOR method to converge are $\operatorname{det}(D-r E) \neq 0$ and either $0<\omega, \max \{\omega, 2\} \leq r$ (the pair $(r, \omega)=(2,2)$ is excluded) or $r \leq \omega<0$.

PROOF. We observe that, in either case, we have $\frac{2-r}{\omega} \leq 0$ and $\frac{r-\omega}{\omega} \geq 0$, where at least one of the inequalities is a strict one. Since the Hermitian matrices $D$ and $A$ are negative and positive definite respectively, then, because of the relationships for $r$ and $\omega$, just obtained, we have from the last expression for $M$ in (2.2) that $M$ is positive definite. The latter implies the convergence of the AOR method.

3. GENERAL COMMENTS, DISCUSSIONS AND FINAL REMARKS.

As has already been seen, the theory presented in the previous section extends not only the theory of the AOR method but also generalizes some well-known results concerning extensions of the SOR method. Thus, in view of what has already been discussed, we are now in a position to clarify some of the points of the previous sections and also to indicate some questions for further research.

i) Examining very carefully the corresponding $\omega$ - and $r$-domains in all the Cases of the table, we can find that their intersection is the empty set except in the cases and subcases where $\omega \in(0,2)$. For example, in the first subcase of Case 1 , we have $(-\infty, 0) \cap\left(\omega+(2-\omega) / \mu_{m},+\infty\right)=\emptyset$ since $\omega<\omega+(2-\omega) / \mu_{m}$ Thus we come to the expected conclusion that, for the generalized SOR method, the Generalized Ostrowski Theorem (Theorem 1) does hold for $\omega \in(0,2)$.

ii) It is astonishing the variety of ways one can use to prove Theorem 1 . For the interested reader, we just outline two of them very briefly. a) If we denote by $\varepsilon_{\mathrm{m}} \mid \mathrm{m}=0,1,2, \ldots$ the error vector of the scheme (1.4) at the $\mathrm{m}^{\text {th }}$ iteration (assuming $\varepsilon_{0} \neq 0$ ), define the quantities $\delta_{\mathrm{m}}=\varepsilon_{\mathrm{m}}-\varepsilon_{\mathrm{m}+1} \mid \mathrm{m}=0,1,2, \ldots$ and follow the steps as in Varga (see [12], pp. 77-78), we arrive at the relationship $\delta_{\mathrm{m}}^{\mathrm{H}} \delta_{\mathrm{m}}=\varepsilon_{\mathrm{m}}^{\mathrm{H}} \mathrm{A} \varepsilon_{\mathrm{m}}-\varepsilon_{\mathrm{m}+1}^{\mathrm{H}}{ }_{\mathrm{m}+1}$. The latter is the same as relationsh1p (3.56) of [12], 
the only difference being that instead of $M$ the reduced form of it corresponding to the generalized SOR method, namely $\left(\frac{2-\omega}{\omega}\right) D$, is present. Consequently, by following the reasoning of [12], the theorem can be proved. b) By following the theory given in Young (see [17], pp. 80-84), in which Lyapunov's theorem is contained, and using $(1.5),(1.6)$ and $(2.2)$, we form the expression $\left(I-L_{r, \omega}\right)^{-1}\left(I+L_{r, \omega}\right)=A^{-1}\left(M-\frac{r}{\omega} S\right)$. It is easy to prove that if $\mathrm{A}$ is positive definite the real parts of the eigenvalues of the matrix $A^{-1}\left(M-\frac{r}{\omega} S\right)$ are positive so that $\rho\left(L_{r, \omega}\right)<1$ and, if $\rho\left(L_{r, \omega}\right)<1$, the real parts of the eigenvalues of $A^{-1}\left(M-\frac{r}{\omega S}\right)$ are positive implying that $A$ is positive definite. It should be noted that the proof just outlined was pointed out (for a similar problem) to the first of the authors by Dr. M. Neumann [20].

iii) There exist other possible proofs for Theorem 2, too. One of them is that based on a straightforward application of Theorem 2.2 of [19], taking into account that $\mathrm{A}$ is Hermitian and positive definite.

iv) Niethammer [4, Theorem 1A] gives the $\omega$ - and $r$-domains for which his Extrapolated SOR method, for $A$ positive definite and $D=\operatorname{diag}(A)=I$, converges. Since he also assumes that $\mu_{m}<0$, his results

$$
0<r \leq 2, \quad 0<\omega<\left(r \mu_{m}-2\right) /\left(\mu_{m}-1\right)
$$

and

$$
2<\mathrm{r}<2 / \mu_{M}, \quad 0<\omega<\left(r \mu_{M}-2\right) /\left(\mu_{M}-1\right)
$$

cover only Case 4 of our table and must coincide with the results given there. However, if we solve for $\omega$ in terms of $r$, and not the other way as we have done when constructing the table, the corresponding domains for our Case 4 can be found to be almost the same as those in (3.1). The only difference is that our LHS for the first series of inequalities for $\mathrm{r}$ is $2 / \mu_{\mathrm{m}}<0$. Thus, our results, even in that specific case, are slightly better. This had to be expected because of two reasons. The first is that the case $r=0$ is not covered in [4] due to the form of the scheme there. The second is that only positive values for the ratio $\alpha=\omega / \mathrm{r}$ are considered. The latter, in view of the fact that $\omega$ happens to be always positive (see (3.1)), has as a consequence that $r$ in Niethammer's case can not be negative. 
v) The problem of finding optimum parameters for $\omega$ and $r$ has been solved so far in two cases only. More specifically, Niethammer [4] solved the problem for the scheme (1.4) with $\mathrm{D}=\operatorname{diag}(\mathrm{A})=\mathrm{I}, \mathrm{A}=\mathrm{I}-\mathrm{B}, \mathrm{B}$ weakly 2-cyclic consistently ordered, and $\rho(B)<1$ in the two cases: a) A Hermitian positive definite and b) B skew-Hermitian. Avdelas and Hadjidimos [21] solved, quite independently, the aforementioned problem (a) by considering the whole $(r, \omega)-p l a n e$ and found the same optimum parameters and, in two very special cases, some better ones. Varga [13] solved the problem for the SOR scheme (1.4) ( $r=\omega)$ with A and D Hermitian positive definite and $\omega \in(0,2)$ and, finally, Hadjidimos [14] did the same with D negative definite and $\omega \in(-\infty, 0) U(2,+\infty)$. Having in mind the complicated analysis, which even the simplest special cases require, we reach the conclusion that the general problem for the AOR scheme (1.4) must be a difficult one. It constitutes an open problem which is being investigated.

vi) The problem of sharpness of some upper bounds for the spectral radius $\rho\left(L_{r, \omega}\right)$, which was put and solved first by Varga [13] in a special case for $r=\omega$ (SOK method) and then by Neumann and Varga [16] in cases for $r=\omega$ and a class of matrices $\mathrm{A}$, is directly connected with the solution of the problem of the optimum parameters mentioned in (v) previously. Thus, this new general problem remains an open one and, at the moment, it can be tackled only in special cases for which the optimum parameters are already available.

vii) A final remark concerning the improvements of the results of this paper by the technique developed recently by de Pillis [15]. In [15], second order iterative schemes from first order ones are constructed by means of an ellipse symmetric to the real and imaginary axes which captures the spectrum $\sigma(B)$ of the iteration matrix $B$ of the first order procedure. The second order schemes, which are constructed under the restriction that $\sigma(B)$ lies in the infinite strip $\{z:|\operatorname{Rez}|<1\}$, are in general faster that the corresponding first order ones. However, as de Pillis remarks in $\$ 5$ of [15] in case the capturing ellipse is a circle, then the second order scheme degenerates into the corresponding first order one. In [21], the idea by de Pillis is exploited further by considering a monoparametric family of captur- 
ing ellipses and among them the one for which the rate of convergence of the second order scheme is a maximum. Thus, in the case of the AOR scheme (1.4) (in fact any AOR scheme can be treated similarly), a combination of the techniques of [21] and [15] may improve the convergence rates. For this, the operator A of the original system (1.1) is written in the form of the multiplicative splitting $A_{0}$ (I - B) (see [15]) with $A_{0}=\frac{1}{\omega}(D-r E)$ and $B=L_{r, \omega}$ and then, assuming knowledge of $\sigma\left(L_{r, \omega}\right)$, the optimum capturing ellipse (see [21]) is found. Unless $\rho\left(\mathrm{L}_{\mathrm{r}, \omega}\right)<1$ and the optimum capturing ellipse is a circle, the rate of convergence of the AOR scheme can not be improved upon by combining the techniques of [15] and [21]; in all the other cases, an improvement can be achieved. It is understood that the optimum pair $(r, \omega)$ must be used in the AOR scheme whenever this is available. ACKNOWLEDGEMENT. .

The authors are most grateful to the referee for useful suggestions and constructive criticism for the improvement of this paper.

\section{REFERENCES}

1. SISLER, M. Über ein zweiparametriges Iterationsverfahrens, Ibid. 18, (1973) $325-332$.

2. SISLER, M. Über die Optimierung eines zweiparametrigen Iterationsverfahrens, Ibid. 20, (1975) 126-142.

3. SISLER, M. Bemerkungen zur Optimierung eines zweiparametrigen Iterationsverfahrens, Ibid. 21, (1976) 213 - 220 .

4. NIETHAMMER, W. On Different Splittings and the Associated Iteration Methods, SIAM J. Numer. Ana1. 16, (1979) $186-200$.

5. HADJIDIMOS, A. Accelerated Overrelaxation Method, Math. Comp. 32 (1978) $149-157$.

6. AVDELAS, G. and HADJIDIMOS, A. Optimum Accelerated Overrelaxation Method in a Special Case, Math. Comp. 36 (1981) 183 - 187.

7. HADJIDIMOS, A. Some Basic Results on M-Matrices in Connection with the Acce1erated Overrelaxation (AOR) Method, Computing 24 (1980) 259 - 268.

8. HADJIDIMOS, A. and YeYIOS, A. The Principle of Extrapolation in Connection with the Accelerated Overrelaxation (AOR) Method, Linear Algebra Appl. 30 (1980) $115-128$.

9. HADJIDIMOS, A. and YEYIOS, A. Symmetric Accelerated Overrelaxation (SAOR) Method, Numer. Math. (under revision). 
10. YEYIOS, A. On the Accelerated Overrelaxation (AOR) Method for Solving Large Linear Systems (Greek), Ph.D. Thesis, University of Ioannina, Ioannina, Greece, (1979).

11. OSTROWSKI, A.M. On the Linear Iteration Procedures for Symmetric Matrices, Rend. Mat. e App1. 13 (1954) 140 - 163.

12. VARGA, R.S. Matrix Iterative Analysis, Prentice-Hall, New Jersey, (1962).

13. VARGA, R.S. Extensions of the Successive Overrelaxation Theory with Applications to Finite Element Approximations in "Topics in Numerical Analysis" (Edit. MILlER J.H.H.), Academic Press, New York (1973) 329 - 343.

14. HADJIDIMOS, A. Three-Part Splittings and Varga's Type Extensions of the Successive Overrelaxation (SOR) Theory, Math. Comput. Simulation XXII (1980) $242-247$.

15. De PILLIS, J. How to Embrace your Spectrum for Faster Iterative Results, Linear Algebra App1. 34 (1980) 125 - 143.

16. NEUMANN, M. and VARGA, R.S. On the Sharpness of Some Upper Bounds for the Spectral Radii of S.0.R. Iteration Matrices, Numer. Math. 35 (1980) $69-70$.

17. YOUNG, D.M. Iterative Solution of Large Linear Systems, Academic Press, New York (1971).

18. WIGNER, E.P. On Weakly Positive Matrices, Canad. J. Math. 15 (1963) 313 - 317.

19. ORTEGA, J.M. and PLEMMONS, R.J. Extensions of the Ostrowski-Reich Theorem for SOR Iterations, Linear Algebra Appl. 28 (1979) 177 - 191.

20. NEUMANN, M. Personal Communication.

21. AVDELAS, G., GALANIS, S. and HADJIDIMOS, A. On the Optimization of a Class of Second Order Iterative Schemes, T.R. No 66, Department of Mathematics, University of Ioannina, Ioannina, Greece (1981). 


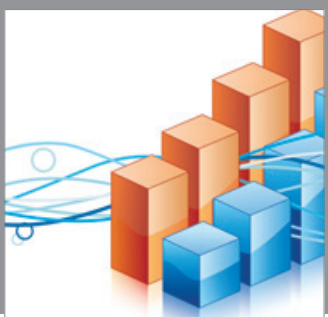

Advances in

Operations Research

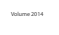

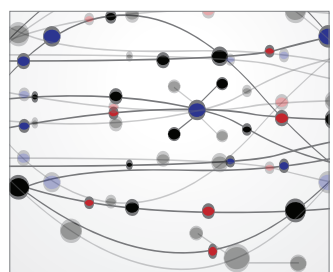

\section{The Scientific} World Journal
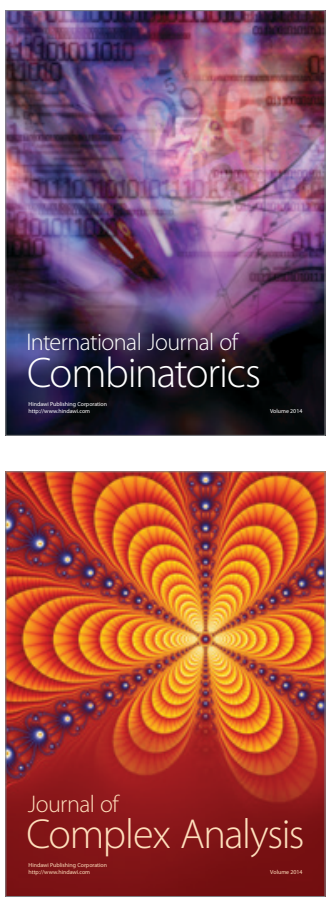

International Journal of

Mathematics and

Mathematical

Sciences
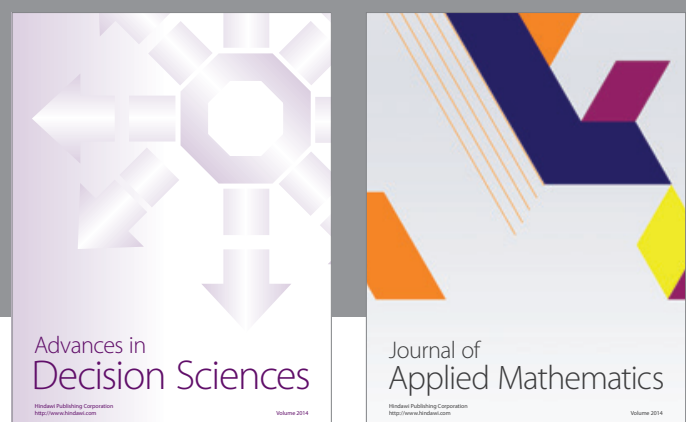

Journal of

Applied Mathematics
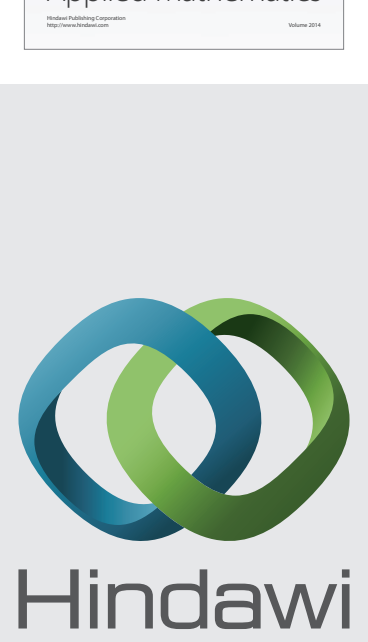

Submit your manuscripts at http://www.hindawi.com
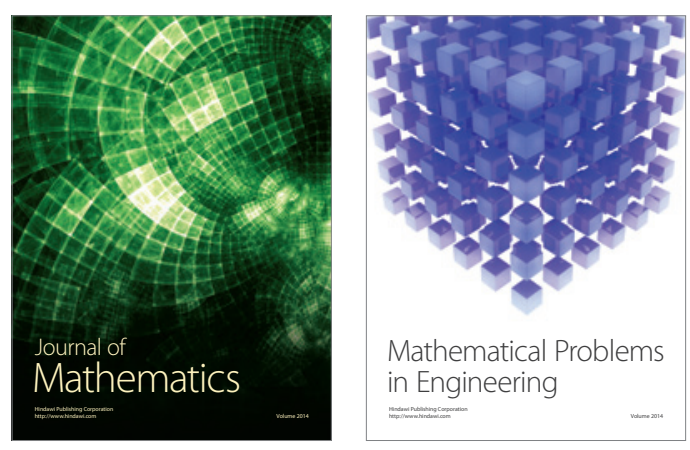

Mathematical Problems in Engineering
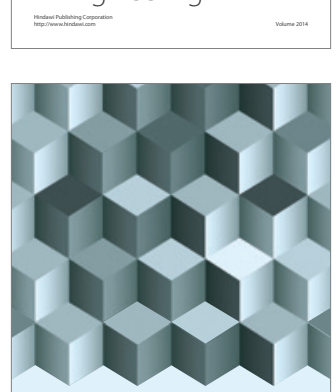

Journal of

Function Spaces
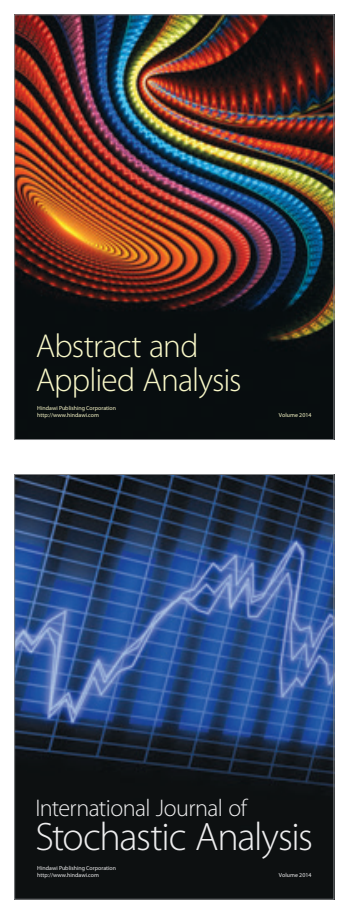

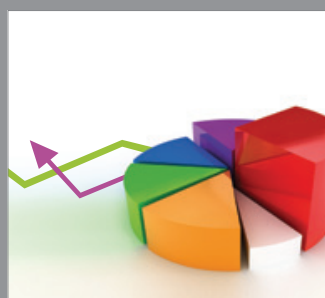

ournal of

Probability and Statistics

Promensencen
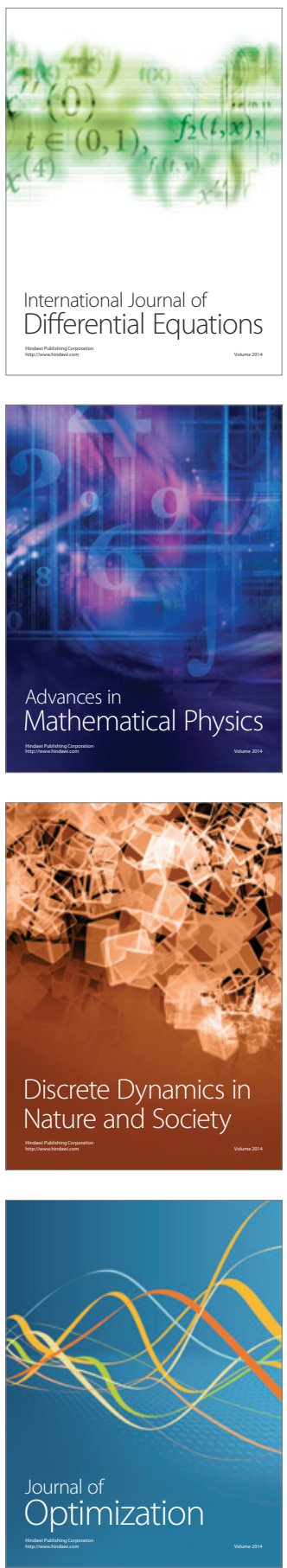